\title{
Hematoma subperiosteal de órbita: relato de caso
}

\author{
Subperiosteal hematoma of theorbit:case report
}

\author{
Jacinto Barbosa Lay Chaves ${ }^{1}$ \\ Marcus Sabry Azar Batista ${ }^{2}$ \\ Ronie Leo Piske ${ }^{3}$ \\ Kaile de Araújo Cunha ${ }^{4}$ \\ Kelson James Silva Almeida ${ }^{5}$
}

\section{RESUMO}

Descrevemos um paciente de 16 anos que após traumatismo crânioencefálico leve evoluiu com dor ocular, hematoma palpebral unilateral e proptose do olho esquerdo. Tomografia computadorizada de órbitas evidenciou hematoma subperiosteal em órbita esquerda. Foi realizada drenagem cirúrgica da coleção. O hematoma subperiosteal de órbita, apesar de raro, deve ser incluído como etiologia de proptose ocular e seu diagnóstico deve ser precoce a fim de evitar a morbidade associada.

Descritores: Hematoma/etiologia; Hemorragia/etiologia; Trauma crânio-cerebral/complicações; Exoftalmia; Doenças orbitárias/etiologia; Relatos de casos [Tipo de publicação]

\section{INTRODUÇÃO}

As lesões hemorrágicas orbitárias podem ser classificadas em hemorragias intra-orbitárias e hematomas subperiosteais ${ }^{(1)}$. O hematoma subperiosteal da órbita é uma entidade clínica infreqüente, mas uma importante causa de exoftalmia unilateral ${ }^{(2)}$. Geralmente resulta da ruptura de vasos sangüíneos e formação de hematoma entre o osso e o periósteo separado ${ }^{(3)}$.

É uma rara complicação do traumatismo crânio-encefálico muito embora também possa ocorrer espontaneamente ou ser decorrente de uma doença sistêmica que favoreça sangramentos; de uma elevação súbita da pressão venosa craniana ou de uma sinusite paranasal ${ }^{(1,4)}$.

O presente artigo visa apresentar as características clínicas e radiológicas de um caso de hematoma subperiosteal da órbita. O objetivo é favorecer o rápido reconhecimento da entidade, para que a intervenção seja mais precoce, a fim de reduzir a incidência de complicações.

\section{RELATO DO CASO}

Descrevemos um paciente, 16 anos, do gênero masculino, que procurou o serviço de emergência em outubro de 2003 após sofrer traumatismo crânioencefálico (TCE) em atividade esportiva. Queixava-se de intensa dor ocular e "inchaço" no olho esquerdo que apareceram e evoluíram rapidamente.

Ao exame, observou-se hematoma na pálpebra superior esquerda e proptose do olho esquerdo (Figura 1A). O fundo de olho mostrava-se normal. Havia evidente limitação da movimentação ocular do olho esquerdo no plano vertical.

Submetido à tomografia computadorizada (TC) de crânio e órbita (Figura 2), foi evidenciada uma lesão hiperdensa de aspecto biconvexo no teto orbitário esquerdo. Realizou-se então orbitotomia que permitiu acesso à coleção sangüínea coagulada, sem pontos sangrantes ativos (Figura 1B). Procedeu-se à drenagem do hematoma subperiosteal orbital (HSPO). O paciente recuperou-se totalmente do quadro clínico descrito após a inter- 


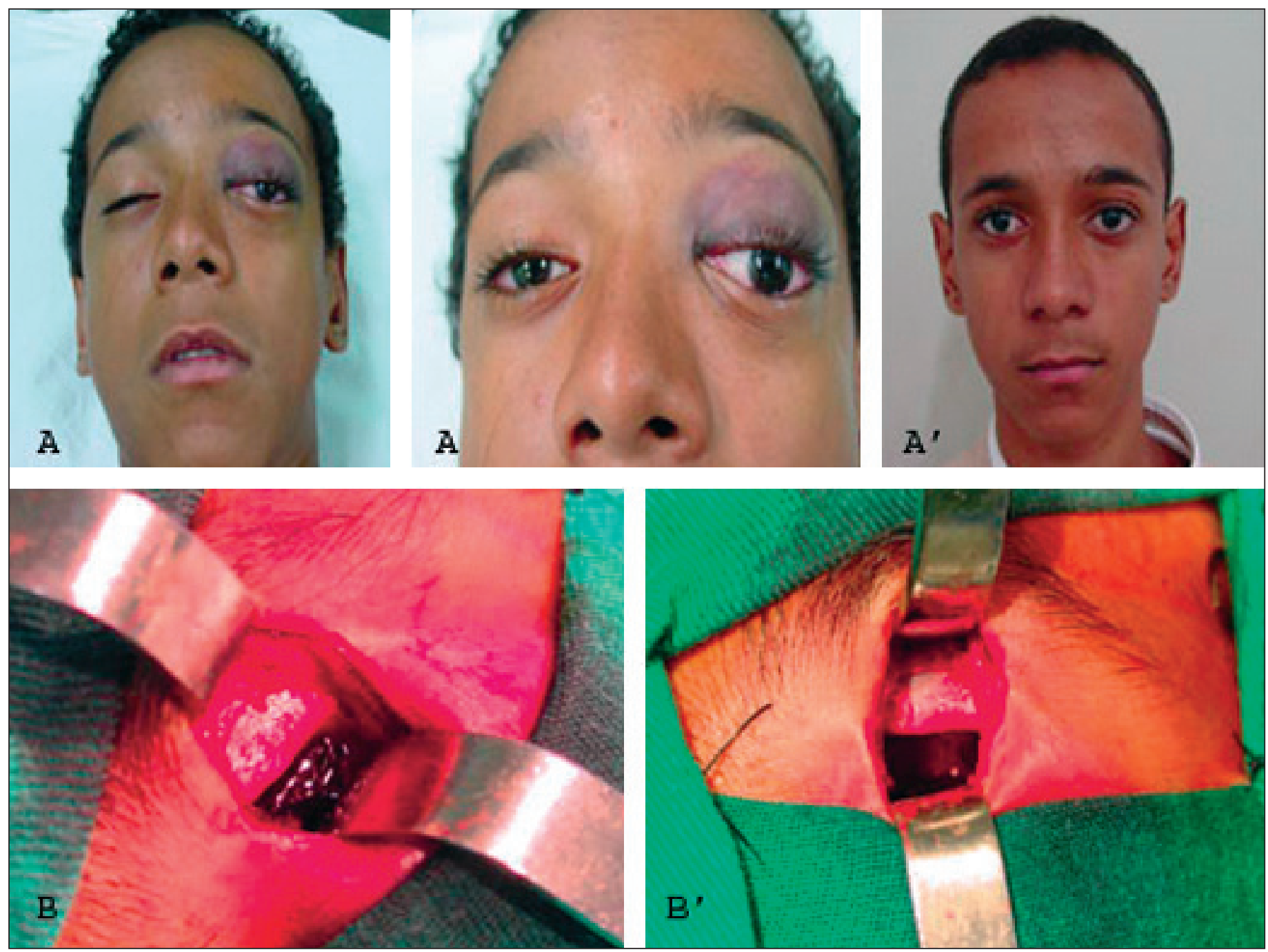

Figura 1 - A: Aspecto clínico pré-operatório; A': Aspecto clínico do paciente no seguimento pós-operatório. B: Orbitotomia evidenciando hematoma subperiosteal da órbita esquerda antes e após (B') a drenagem cirúrgica.

venção cirúrgica. Durante seguimento ambulatorial foi realizada TC de controle que se mostrou normal.

\section{DISCUSSÃO}

Os HSPO são raros e geralmente ocorrem logo após TCE, muito embora possam decorrer meses ou anos para o seu aparecimento; o trauma pode ser mínimo ou não haver história bem definida de trauma ${ }^{(4)}$. Acometem quase exclusivamente crianças e adultos jovens, localizando-se principalmente no teto orbitário em sua porção de osso frontal ${ }^{(4-5)}$.

$\mathrm{O}$ osso frontal forma a maior concavidade da superfície orbitária. O seu periósteo, principalmente em crianças e adultos jovens, não estaria firmemente aderido, exceto nas linhas de sutura, fator que predispõe ao surgimento potencial de hematomas nessa região, nessa faixa etária. Sabe-se que a adesão entre periósteo e osso aumenta com a idade ${ }^{(4-5)}$.

A patogênese do HSPO decorre de um sangramento locali- zado no espaço subperiosteal da órbita por trauma ou secundária à transmissão de um aumento de pressão na rede venosa adjacente ${ }^{(2)}$. Nos casos de sinusite associada, a flebite na mucosa do seio paranasal pode se estender para as veias peri-orbitárias, resultando em ruptura subseqüente desses $\operatorname{vasos}^{(6-7)}$.

Há diversas causas implicadas no surgimento do HSPO. Dentre elas podemos citar: trauma ${ }^{(2,4,6,8-10)}$, congestão venosa (tosse grave, manobra de Valsava, trabalho de parto) ${ }^{(1,11)}$, discrasias sangüíneas (anemia falciforme, $\beta$-talassemia, leucemia, coagulopatias) ${ }^{(5,10,12)}$, sinusite para-nasal crônica ${ }^{(6-7)}$, HSPO associado a hematoma extradural e subdural ${ }^{(11,13-14)}$, idiopática (HSPO espontâneo, o qual geralmente acomete indivíduos mais idosos da faixa etária 43 a 81 anos). Acredita-se que alguns distúrbios vasculares associados à idade, como arteriosclerose, microaneurismas e instabilidade vasomotora, possam ser causas de HSPO espontâneo ${ }^{(1-2)}$.

O quadro clínico (Tabela 1) caracteriza-se geralmente por início súbito de exoftalmia proeminente, dor ocular intensa, deslocamento ínfero-lateral do globo ocular, dificuldade na 

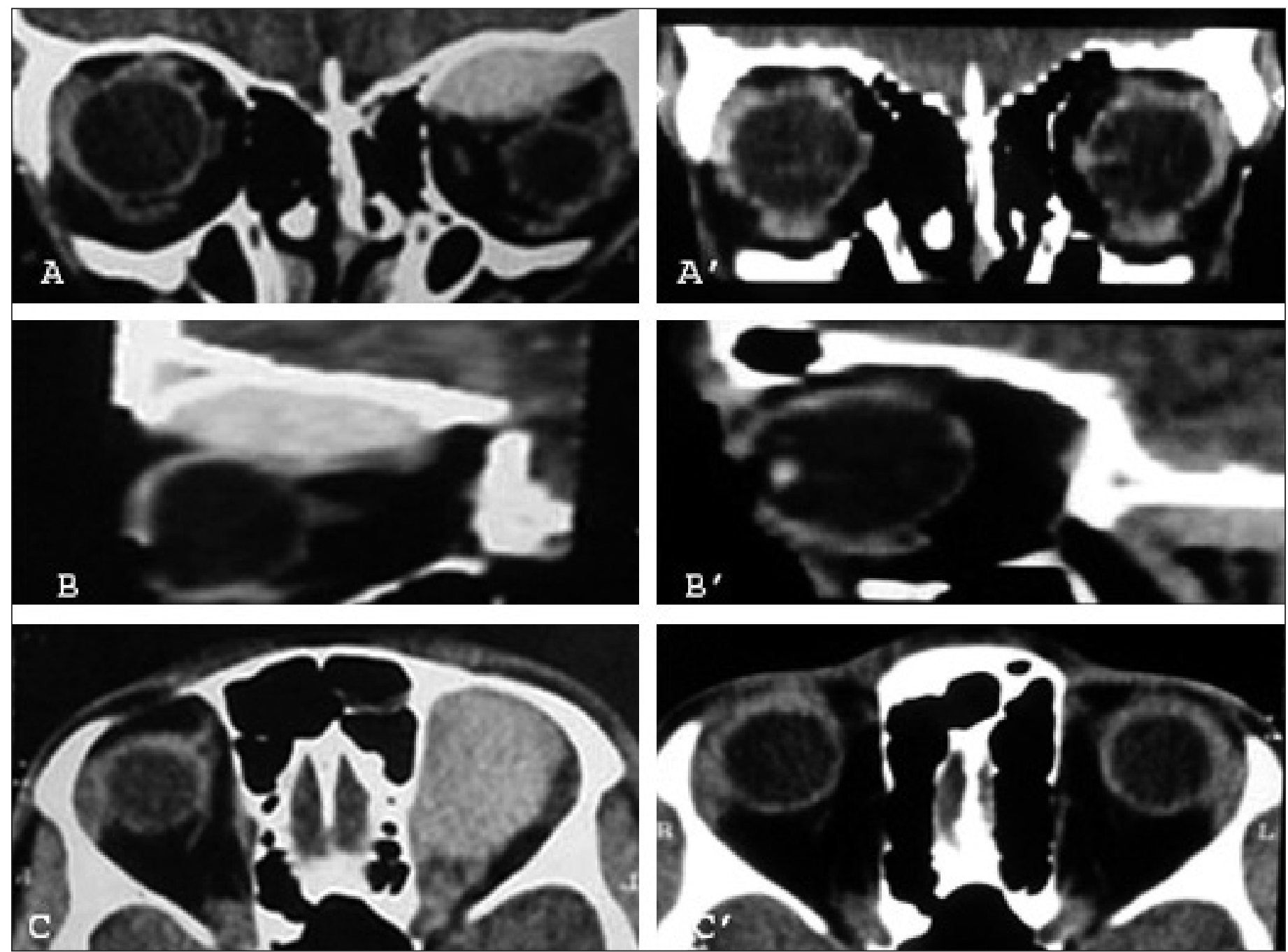

Figura 2 - A, B, C: Tomografia computadorizada evidenciando hematoma subperiosteal de órbita esquerda. A', B', C': Aspecto radiológico de controle no pós-operatório.

movimentação da musculatura ocular extrínseca e alterações da acuidade visual podendo evoluir até a cegueira. $\mathrm{O}$ exame oftalmológico evidencia diversos sinais, sendo os principais: aumento da pressão intra-ocular, papiledema e congestão venosa, hemorragia sub-retinal ${ }^{(2-4,15)}$.

O diagnóstico precoce é importante, pois, diante do quadro clínico e com características radiológicas específicas, o cirurgião pode descomprimir precocemente a órbita, evitando seqüelas graves tais como cegueira, atrofia do nervo óptico, estrabismo secundário, ou até mesmo exoftalmia persistente devido à fibrose e calcificação do hematoma ${ }^{(4)}$. Para melhor avaliação pré-cirúrgica, pode ser necessária a realização de arteriografia, TC de órbita ou ressonância nuclear magnética (RNM).

A arteriografia demonstra uma massa avascular supra-orbitária com estreitamento e deslocamento inferior da artéria oftálmica ipsilateral ${ }^{(2)}$. A ausência de anomalias venosas orbitárias ajuda a distinguir pacientes com HSPO traumático daqueles com hemorragia orbitária espontânea. A arteriografia também é útil no diagnóstico diferencial de fístula carótido-cavernosa ${ }^{(4)}$.
A TC de órbita com reconstruções sagitais e coronais permite o delineamento preciso do tamanho e extensão do HSPO. Possui as seguintes vantagens: a) define nitidamente uma massa não captante de contraste, hiperdensa, biconvexa com ampla base de implantação principalmente na porção superior do teto orbitário; b) permite a observação do HSPO em relação às outras estruturas vizinhas: tecidos moles, músculos conais, nervo óptico e globo ocular, e ainda mostra deslocamento inferior do conteúdo orbitário; c) demonstra estreitamento do nervo óptico ${ }^{(2,4)}$.

RNM é o exame de imagem ideal por ter capacidade de identificar sangue e produtos hemorrágicos em todos os estágios de degradação ${ }^{(16)}$. Demonstra uma massa extraconal biconvexa localizada na parte superior da órbita com diferentes graus de intensidade de sinal em T1 e T2, dependente do estado de degradação dos componentes sangüíneos ${ }^{(2)}$.

Conforme mencionado, as lesões hemorrágicas orbitárias podem ser classificadas em intra-orbitárias e subperiosteais. Aquelas são mais comuns e determinam achados como: equimo- 


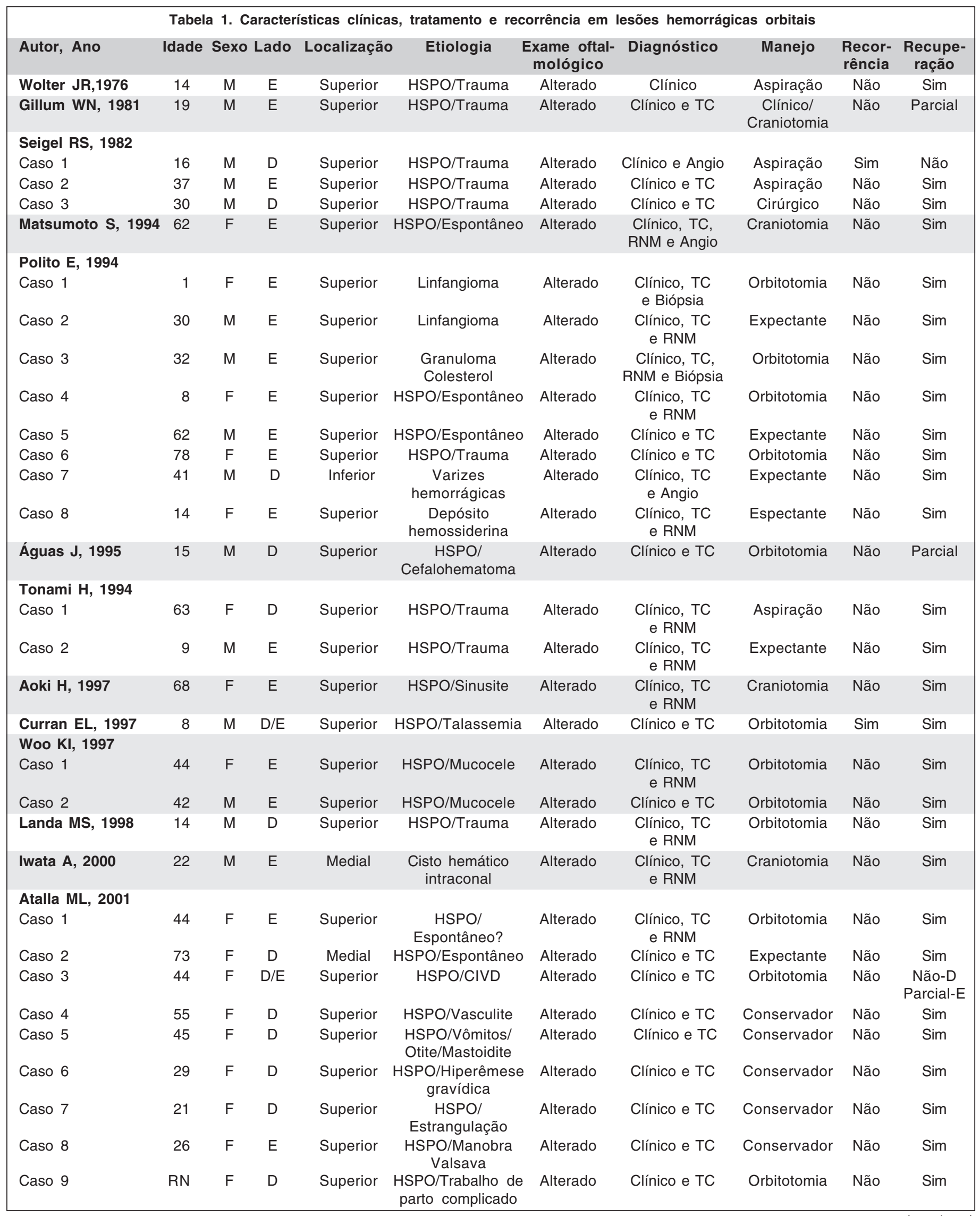




\begin{tabular}{|c|c|c|c|c|c|c|c|c|c|c|}
\hline Autor, Ano & Idade & Sexo & Lado & Localização & Etiologia & $\begin{array}{l}\text { Exame oftal- } \\
\text { mológico }\end{array}$ & Diagnóstico & Manejo & $\begin{array}{l}\text { Recor- } \\
\text { rência }\end{array}$ & $\begin{array}{l}\text { Recupe- } \\
\text { ração }\end{array}$ \\
\hline Rojas MC, 2002 & 9 & $\mathrm{~F}$ & D & Superior & HSPO/Trauma & Alterado & Clínico e TC & Orbitotomia & Não & Sim \\
\hline Sabet SJ, 2001 & 9 & M & D & Superior & HSPO/Trauma & Alterado & Clínico e TC & Orbitotomia & Não & Sim \\
\hline Naja A, 2002 & 16 & $M$ & E & Superior & HSPO/Trauma/HED & Alterado & Clínico e TC & Craniotomia & Não & Sim \\
\hline Amlashi SF, 2003 & 29 & $M$ & D & Superior & HSPO/Trauma & Alterado & Clínico e TC & Craniotomia & Não & Sim \\
\hline Choi HY, 2004 & 44 & $\mathrm{~F}$ & $E$ & Superior & $\begin{array}{l}\text { HSPO/ latrogenia/ } \\
\text { Microcirurgia } \\
\text { Aneurisma }\end{array}$ & Alterado & Clínico e TC & Orbitotomia & Não & Sim \\
\hline
\end{tabular}

se e/ou edema palpebral, deslocamento axial do globo ocular, hemorragia conjuntival difusa, motilidade ocular restrita e variados graus de disfunção visual. Estas se caracterizam por proptose, deslocamento ínfero-lateral do globo ocular, ausência de equimose palpebral, dificuldade na movimentação da musculatura ocular extrínseca e variados graus de disfunção visual ${ }^{(5)}$.

O diagnóstico diferencial das lesões hemorrágicas orbitárias pode ser difícil pela sua relativa raridade e apresentação inespecífica. Incluem um grupo heterogêneo de condições idiopáticas ou causadas por traumas, cirurgias, tumores ou malformações vasculares pré-existentes ${ }^{(16)}$. Os principais diagnósticos diferenciais são: linfangiomas císticos, hematoma traumático, varizes hemorrágicas, granuloma de colesterol, tumores orbitários, depósito de hemossiderina pós-cirúrgico, mal-formações arteriovenosas, fístula carótido-cavernosa ${ }^{(5,16-17)}$.

O tratamento do HSPO depende da gravidade da compressão orbitária e da natureza da doença associada ${ }^{(2,7,15,17)}$. Há relatos de regressão espontânea ${ }^{(16)}$. No entanto, o tratamento deve ser conduzido da seguinte forma:

- Em pacientes em que a quantidade de hematoma é insigni-

ficante e a acuidade visual não está comprometida, obser-

vação e seguimento são recomendados ${ }^{(5,7,17)}$,

- Nos pacientes que apresentam déficit visual, dor ocular intensa e doenças associadas (por exemplo: sinusite), a exploração cirúrgica está indicada ${ }^{(5,7)}$.

Há três modalidades cirúrgicas principais: aspiração por agulha, orbitotomia infraciliar e craniotomia com acesso ao teto orbitário pela fossa craniana anterior. Apesar de a aspiração por agulha do HSPO ser tecnicamente mais simples, a orbitotomia apresenta vantagens uma vez que permite a remoção completa do coágulo, a correção de uma possível fratura nos ossos da órbita, hemostasia de vasos sangrantes reduzindo o risco de novas hemorragias e permite colocação de dreno. Já craniotomia é tão efetiva quanto à orbitotomia, possuindo a desvantagem de ser mais invasiva ${ }^{(2)}$.

O HSPO deve ser incluído como diagnóstico diferencial das causas de proptose ocular. Sua identificação precoce possibilita a intervenção precoce e um prognóstico mais favorável.

\section{ABSTRACT}

We describe a sixteen years old male patient that suffered a craniocerebral trauma and presented at the emergency room, with ocular pain, palpebral hematoma and left eye proptosis. Orbital computed tomography showed left subperiosteal orbital hematoma. A surgical drainage was performed. Subperiosteal hematoma of the orbit is a rare cause of proptosis; its early diagnosis must be made in order to avoid possible complications.

Keywords: Hematoma/etiology; Hemorrhage/etiology; Craniocerebral trauma/complications; Exophthalmos; Orbital diseases/etiology; Case reports [Publication type]

\section{REFERÊNCIAS}

1. Wolter JR, Leenhouts JA, Coulthard SW. Clinical picture and management of subperiosteal hematoma of the orbit. J Pediatr Ophthalmol. 1976;13(3):136-8.

2. Gillum WN, Anderson RL. Reversible visual loss in subperiosteal hematoma of the orbit. Ophthalmic Surg. 1981;12(3):203-9.

3. Matsumoto S, Yamamoto T, Ban S, Motozaki T, Sato S, Shingu T, et al. Spontaneous subperiosteal hematoma of the orbit - case report. Neurol Med Chir (Tokyo). 1994;34(1):27-9.

4. Seigel RS, Williams AG, Hutchison JW, Wolter JR, Carlow TJ, Rogers DE. Subperiosteal hematomas of the orbit: angiographic and computed tomographic diagnosis. Radiology.1982;143(3):711-4.

5. Woo KI, Kim YD. Subperiosteal hematoma of the orbit associated with sinusitis. Korean J Ophthalmol. 1997;11(2):118-22.

6. Tonami H, Kuginuki Y, Okimura T, Yamamoto I, Kawakami S, Sasaki K. MRI of subperiosteal hematoma of the orbit. J Comput Assist Tomogr. 1994; 18(4):549-51.

7. Landa MS, Landa EH, Levine MR. Subperiosteal hematoma of the orbit: case presentation. Ophthal Plast Reconstr Sur. 1998;14(3):189-92.

8. Polito E, Leccisotti A. Diagnosis and treatment of orbital hemorrhagic lesions. Ann Ophthalmol. 1994;26(3):85-93.

9. Aguas J, Conde C, Fructuoso GG, Mondelo FJ, Ferrer E. Giant cephalhematoma in a 15-year-old boy. Unilateral amaurosis as the main complication. Surg Neurol. 1995;43(4):363-6.

10. Aoki H, Tanaka Y, Niki Y, Kamada K, Fujita T. Intraorbital subperiosteal hematoma due to paranasal mucocele - case report. Neurol Med Chir (Tokyo). 1997;37(8):627-9.

11. Curran EL, Fleming JC, Rice K, Wang WC. Orbital compression syndrome in sickle cell disease. Ophthalmology. 1997;104(10):1610-5.

12. Marback EF, Marback PMF, Marback RL, Sampaio CM, Sé DCS. Síndrome de compressão orbitária relacionada à anemia falciforme. Arq Bras Oftalmol. 1999;62(5):631-4.

13. Iwata A, Matsumoto T, Mase M, Yamada K. Chronic, traumatic intraconal hematic cyst of the orbit removed through the fronto-orbital approach - case report. Neurol Med Chir (Tokyo). 2000;40(2):106-9.

14. Atalla ML, McNab AA, Sullivan TJ, Sloan B. Nontraumatic subperiosteal orbital hemorrhage. Ophthalmology. 2001;108(1):183-9. Review.

15. Rojas MC, Eliason JA, Fredrick DR. Needle aspiration of a traumatic subperiosteal haematoma of the orbit. Br J Ophthalmol. 2002;86(5):593-4.

16. Sabet SJ, Tarbet KJ, Lemke BN, Smith ME, Albert DM. Subperiosteal hematoma of the orbit with osteoneogenesis. Arch Ophthalmol. 2001;119(2):301-3.

17. Amlashi SF, Riffaud L, Guyomard JL, Brassier G, Morandi X. [Post-traumatic exophthalmos caused by a subperiosteal hematoma of the orbit]. Neurochirurgie. 2003;49(2-3 Pt 1):107-9. French. 